\title{
Cross-Cultural Translation and Validation of the Spanish Version of the Patellofemoral Pain and Osteoarthritis Subscale of the KOOS (KOOS-PF)
}

Juan Pablo Martinez-Cano ( $\sim$ jpmartinezc@gmail.com )

Departamento de Ortopedia y Traumatología, Cra https://orcid.org/0000-0002-6228-0621

Daniel Vernaza-Obando

Universidad ICESI Facultad de Ciencias de la Salud

Julián Chica

Fundacion Valle del Lili

Andrés Mauricio Castro

Fundacion Valle del Lili

\section{Research}

Keywords: Patient Reported Outcome Measures, Patellofemoral Pain Syndrome, Validation Study, Translating.

Posted Date: January 13th, 2021

DOI: https://doi.org/10.21203/rs.3.rs-141893/v1

License: (c) (i) This work is licensed under a Creative Commons Attribution 4.0 International License. Read Full License 


\section{Abstract}

Background: Patellofemoral pain is a very common complaint in orthopedic sports medicine clinics. Disease-specific patient-reported outcome measures (PROMs) are useful for research and clinical practice; thus, it is important to have validated translations available for new PROMs. This study aims to translate and validate the Spanish version of the patellofemoral pain and osteoarthritis subscale of the knee injury and osteoarthritis outcome score (KOOS-PF).

Methods: The KOOS-PF was translated and culturally adapted to Spanish following current guidelines, which included translation, back-translation, conciliation and pilot studies. Patients with a diagnosis of patellofemoral pain and/or osteoarthritis from one medical center were invited to participate and complete the questionnaire. The evaluation of the score included internal consistency (Cronbach's alpha), floor and ceiling effects, measurement error, minimal detectable change and minimal important change. For test-retest reliability, the intraclass correlation coefficient (ICC) was used, and for responsiveness, the global rating of change (GROC) scale was measured one month later.

Results: Sixty patients with patellofemoral pain and/or osteoarthritis were included in the study. The Spanish version showed very good internal consistency (Cronbach's alpha: 0.93) and test-retest reliability (intraclass correlation coefficient: 0.82). Responsiveness was confirmed, with the KOOS-PF demonstrating a strong correlation with the GROC score ( $r$ 0.64). The minimal detectable change was 11.1 points, the minimal important change was 17.2 points, and there were no floor or ceiling effects for the score.

Conclusions: The Spanish version of the KOOS-PF demonstrated very good measurement properties, including internal consistency, reliability and responsiveness. The KOOS-PF can be used in Spanishspeaking patients for clinical and research purposes in patellofemoral pain and osteoarthritis.

Trial registration: Fundación Valle del Lili, Biomedical Research Ethical Committee: No. 01438.

\section{Background}

Patellofemoral pain is usually located around the kneecap and increases with squats and climbing stairs, among other activities, such as running ${ }^{1,2}$. Patellofemoral pain is a frequent common in the young active population, affecting one in every four athletes and 2.5 million college students every year in the USA ${ }^{3,4}$. It is also the most common cause of consultation in sports medicine clinics and represents $25 \%$ of knee problems ${ }^{4,5}$. Patellofemoral pain may be part of a degenerative joint disorder in middle-aged and elderly populations with patellofemoral osteoarthritis.

There is a lack of objective disease-specific scores for patellofemoral pain and osteoarthritis. The Kujala score was the first score developed for patellofemoral pain and has been translated to several languages, including Spanish. However, there have been some criticisms of the Kujala score regarding some technical and difficult-to-understand questions for some patients. Thus, it is desirable to have additional 
scores that may help clinicians evaluate patients with patellofemoral pain. In 2018, Crossley et al developed and published the patellofemoral pain and osteoarthritis subscale KOOS-PF ${ }^{6}$, a selfadministered score that measures pain, stiffness and quality of life in relation to patellofemoral pain and osteoarthritis. The KOOS-PF is a useful tool for evaluating patients with this syndrome in daily clinical practice and for measuring outcomes.

Only one translation of the KOOS-PF score has been published at this point (Arabic), but there are several studies currently on-going. The purpose of this study was to cross-culturally adapt and translate the KOOS-PF score to Spanish and to validate this version. It is important to be able to properly use this disease-specific score in patients with patellofemoral pain syndrome and osteoarthritis in whom the native language spoken is Spanish.

\section{Methods}

This was a validation study for the Spanish version of the KOOS-PF score following the COSMIN (Consensus-based Standards for the selection of health Measurement Instruments) guidelines ${ }^{7}$. It was conducted in two parts: i) Subscale translation to Spanish and ii) Evaluation of measurement properties.

\section{Translation and cross-cultural adaptation}

The authors of the subscale were contacted, and permission was obtained to translate the KOOS-PF subscale to Spanish. The original version includes 11 self-assessment questions grouped into three different categories: stiffness, pain and quality of life. These questions have five possible answers, represented by a box that the participant should tick. Each answer has a numeric value from 0 to 4 . The final score is on a 0-100 scale, where 0 is the worst score and 100 is the best health score.

The process of translation followed the recommendations by Beaton et al. ${ }^{8}$. There were four persons involved in the translation process. Two independent forward translations were performed from the original English version by an orthopedic surgeon (JPMC) fluent in English whose native language was Spanish and a professional language translator. The two versions were then unified and conciliated. This version was then back-translated by two native English-speaking persons, a medical doctor and a professional language translator. They were not familiar with the original English version of KOOS-PF. A final conciliation between the translators with both versions was performed to obtain a final Spanish version that was tested in a pilot group of five patients with patellofemoral pain syndrome. This test allowed us to evaluate the understanding of the questions by the patients and to identify any problems in answering the items. The Spanish version of the KOOS-PF is available free of charge from http://www.koos.nu.

\section{Evaluation of measurement properties}

The measurement properties that the KOOS-PF subscale measured included reliability (internal consistency, test-retest reliability and measurement error), responsiveness and interpretability (smallest 
detectable change (SDC), minimal important change (MIC), minimal important difference (MID) and floor and ceiling effects).

\section{Internal consistency}

Using baseline KOOS-PF data, we calculated Cronbach's alpha, with values between 0.7 and 0.95 considered to be adequate ${ }^{9}$. A lower value suggests poor correlation among subscale items and limits the interpretability of the total overall score. A very high value may suggest item redundancy.

\section{Test-rest reliability}

The intraclass correlation coefficient (ICC) was used to evaluate reliability. For this calculation, patients were asked to answer the score again 7 days after the baseline measurement, and the reliability between those two tests was calculated. Values $\geq 0.7$ were considered adequate ${ }^{9}$. We also calculated the standard error of the mean (SEM), which is equivalent to SD $x \sqrt{ } 1-$ ICC (where SD is the standard deviation of the observed scores) ${ }^{10}$. Finally, we used a Bland-Altman plot to confirm homoscedasticity ${ }^{11}$.

\section{Responsiveness}

To evaluate responsiveness, we used the global rating of change (GROC) score, which is a single-item questionnaire where the patient had five possible options to answer according to their change in knee pain one month after the baseline measurement. The five response options (Likert scale) ranged from 'much worse' (score of 0 ), 'slightly worse' (score of 1), 'about the same' (score of 2), 'slightly better' (score of 3), to 'much better' (score of 4). Patients were offered physical therapy during this month to evaluate responsiveness to this type of treatment. Not all patients took part in the physical therapy.

\section{Convergent validity}

We evaluated the correlation between the KOOS-PF and the Spanish version of the Kujala score ${ }^{12}$. The Kujala score consists of 13 items relating to symptoms and activities associated with anterior knee pain. Each item has between 3 and 5 possible answers, with some items scoring between $0-5$ points and others scoring $0-10$ points. The final score is in the range of $0-100$, where 100 represents perfect health. We used Pearson's correlation coefficients to evaluate convergent validity.

\section{Interpretability}

We defined floor and ceiling effects as $15 \%$ or more of the sample scoring the lowest or highest possible score on the KOOS-PF. The smallest detectable change was estimated at $90 \%$ confidence (SDC90), for individual changes as $1.65 \times \sqrt{ } 2 \times \mathrm{SEM}$, and for group changes as $1.65 \times \sqrt{2} \times \mathrm{SEM} / \sqrt{ } \mathrm{n}^{13}$.

The minimal important change (MIC) was estimated using the mean KOOS-PF change in score between the baseline and 1-month measurements, for patients reporting to be 'slightly better' 1 month later, using the GROC (score of 3). Meanwhile, the minimal important difference (MID) was estimated as the 
difference in mean change scores between patients reporting being 'slightly better' and those feeling 'about the same' (score of 2$)^{13}$.

\section{Results}

\section{Study participants}

Between June 2019 and March 2020, sixty patients with patellofemoral pain/osteoarthritis were included in the study and completed the Spanish version of the KOOS-PF at baseline. Seven days later, 58 patients completed the test-retest evaluation again. Finally, 55 patients answered the KOOS-PF again at the 30-day follow-up to evaluate responsiveness and meaningful changes. Table 1 shows the baseline characteristics of the participants. The mean age was 31 years old, with an age range from 12-64 years old. Most were women (75\%), and most had pain in both knees (41\%). The mean score (SD) for the KOOS-PF at baseline was 46 (23), and at the 30-day follow-up, it was 61 (24).

Table 1

Baseline characteristics of the study participants with patellofemoral pain in this study.

\begin{tabular}{|ll|}
\hline Variables & $\mathbf{n}=60$ \\
\hline Age, mean \pm SD & $30.95 \pm 10.7$ \\
\hline Sex, $\mathbf{n}(\%)$ & \\
\hline Female & $45(75)$ \\
\hline Male & $15(25)$ \\
\hline Knee, $\mathbf{n}(\%)$ & \\
\hline Right & $17(28)$ \\
\hline Left & $16(27)$ \\
\hline Both & $27(45)$ \\
\hline
\end{tabular}

\section{Internal consistency}

The internal consistency was very high, with a Cronbach's alpha of 0.93 .

\section{Test-retest reliability}

For participants who completed both the baseline and retest questionnaires within 1 week $(n=58)$, the score demonstrated very high test-retest reliability, with an ICC of 0.82 (95\% Cl: 0.697-0.902) and an SEM of 3.7. The Bland-Altman plot showed no systematic differences between the first and second measurements for the KOOS-PF score (Fig. 1). 


\section{Responsiveness}

The KOOS-PF subscale change scores showed a good correlation with GROC scores $(r=0.64)$ (Fig. 2).

\section{Convergent validity}

The Spanish subscale KOOS-PF showed a strong positive correlation with the Spanish version of the Kujala score $(r=0.71)$. Figure 3 shows the scatter plot for this correlation.

\section{Interpretability}

There were no floor or ceiling effects for the KOOS-PF. The individual SDC90 was 8.6, and for the group, the SDC90 was 1.1. The MIC was 17.2, and the MID was 11.1. There were no ceiling or floor effects.

\section{Discussion}

The present study shows the process for translation and validation of the Spanish version of the KOOSPF subscale (Additional file 1). This questionnaire showed strong psychometric properties, such as high reliability and internal consistency. The KOOS-PF also had a strong correlation with similar scores, such as the anterior knee pain Kujala score, and a good responsiveness correlation with the GROC score. These findings are very similar to what was shown with the original English version of the KOOS-PF subscale ${ }^{6}$.

Additionally, similar results were found in a validation of the scale translated to Arabic. While our internal consistency was very good, with a Cronbach's alpha of 0.93 , Ateef ${ }^{14}$ reported a Cronbach's alpha of 0.81 . However, regarding reliability, the Arabic translation had an ICC of $0.96^{14}$, which was better than our ICC of 0.82 . This difference in the intraclass correlation coefficient could be explained by the timing between the first and second questionnaires being administered to participants, with intervals of 48 hours in the former study and 7 days in the latter one. Similarly, none of the translations found floor or ceiling effects.

This study follows the COSMIN checklist for cross-cultural validation ${ }^{7}$. The results indicate that the Spanish version of the KOOS-PF questionnaire is a reliable, valid and responsive measurement tool for use in patients with patellofemoral pain and osteoarthritis. In terms of the smallest detectable change, the score was able to detect very small changes for the whole group (1.1), with still a low number for the individual-level changes (8.6). These values are even smaller than for the original score ${ }^{6}$.

The KOOS-PF showed minimal important differences and minimal important change values that were very similar to the English score values. These data are important for the interpretation of future studies regarding the evaluation of treatments for patellofemoral pain or osteoarthritis. It is very important for clinicians to understand how much difference or change is clinically important for patients.

This is the second validation for a translation of the KOOS-PF subscale. This type of translation and validation permits clinicians and researchers to develop studies using a valid score in patients with patellofemoral pain. 
A limitation of the study might be that the second questionnaire was administered within one week of the baseline questionnaire, during which time some participants could start physical therapy, and some of their symptoms would improve. However, Crossley et al ${ }^{6}$ administered the second questionnaire within two weeks of the first questionnaire. Generally, it is considered that one week is enough time to prevent recall and not enough time to see improvements. In addition, our very good ICC shows that this difference did not affect the results in a considerable way.

\section{Conclusions}

The Spanish version of the KOOS-PF demonstrated very good measurement properties, including internal consistency, reliability and responsiveness. The KOOS-PF can be used in Spanish-speaking patients for clinical and research purposes in patellofemoral pain and osteoarthritis.

\section{Declarations}

Ethics approval and consent to participate: Fundación Valle del Lili, Biomedical Research Ethical Committee: No. 01438. Consent was waived.

Consent for publication: not applicable.

Availability of data and materials: The datasets used and/or analysed during the current study are available from the corresponding author on reasonable request.

Competing interests: The authors declare that they have no competing interests.

Funding: no funding.

Authors' contributions: JPMC: original idea, study design, recruitment and data analysis. DVO: study design and data collection. JC: recruitment, data collection and follow-up. AMC: study design, data analysis. All authors read and approved the final manuscript.

Acknowledgements: not applicable.

\section{References}

1. Dutton RA, Khadavi MJ, Fredericson M. Patellofemoral Pain. Phys Med Rehabil Clin N Am. 2016;27:31-52. doi:10.1016/j.pmr.2015.08.002.

2. Vora M, Curry E, Chipman A, Matzkin E, Li X. Patellofemoral pain syndrome in female athletes: A review of diagnoses, etiology and treatment options. Orthop Rev (Pavia). 2018;9(7281):98-104. doi:10.4081/or.2017.7281.

3. Pappas E, Wong-Tom WM. Prospective Predictors of Patellofemoral Pain Syndrome: A Systematic Review With Meta-analysis. Sports Health. 2012;4(2):115-20. doi:10.1177/1941738111432097. 
4. Devereaux MD, Lachmann SM. Patello-femoral arthralgia in athletes attending a Sports Injury Clinic. Br J Sports Med. 1984;18(1):18-21. doi:10.1136/bjsm.18.1.18.

5. Baquie P, Brukner P. Injuries presenting to an Australian sports medicine centre: a 12-month study. Clin J Sport Med. 1997;7(1):28-31.

6. Crossley KM, Macri EM, Cowan SM, Collins NJ, Roos EM. The patellofemoral pain and osteoarthritis subscale of the KOOS (KOOS-PF): development and validation using the COSMIN checklist. $\mathrm{Br} \mathrm{J}$ Sport Med. 2018;52(17):1130-6. doi:10.1136/bjsports-2016-096776.

7. Mokkink LB, Terwee CB, Knol DL, et al. The COSMIN checklist for evaluating the methodological quality of studies on measurement properties: A clarification of its content. BMC Med Res Methodol. 2010;10(22). doi:10.1186/1471-2288-10-22.

8. Beaton DE, Bombardier C, Guillemin F, Ferraz MB. Guidelines for the process of cross-cultural adaptation of self-report measures. Spine (Phila Pa 1976). 2000;25(24):3186-91. doi:10.1097/00007632-200012150-00014.

9. Terwee CB, Bot SDM, de Boer MR, et al. Quality criteria were proposed for measurement properties of health status questionnaires. J Clin Epidemiol. 2007;60(1):34-42.

doi:10.1016/j.jclinepi.2006.03.012.

10. de Vet HC, Terwee CB, Ostelo RW, Beckerman H, Knol DL, Bouter LM. Minimal changes in health status questionnaires: Distinction between minimally detectable change and minimally important change. Health Qual Life Outcomes. 2006;4(54). doi:10.1186/1477-7525-4-54.

11. Bland JM, Altman DG. Statistical methods for assessing agreement between two methods of clinical measurement. Lancet. 1986;1(8476):307-10.

12. Martínez-Cano JP, Arango AS, Castro AM, Piña AM, Martínez-Rondanelli A. Validación de la escala de Kujala para dolor patelofemoral en su versión en español. Ces Med. 2017;31(1):47-57. doi:https://doi.org/10.21615/ces\%20med.v31i1.3977.

13. Beard DJ, Harris K, Dawson J, et al. Meaningful changes for the Oxford hip and knee scores after joint replacement surgery. J Clin Epidemiol. 2015;68(1):73-9. doi:10.1016/j.jclinepi.2014.08.009.

14. Ateef M. Measurement properties of the knee injury and osteoarthritis outcome score patello-femoral questionnaire in Saudi Arabians. PeerJ. 2020;8. doi:10.7717/peerj.9323.

\section{Figures}




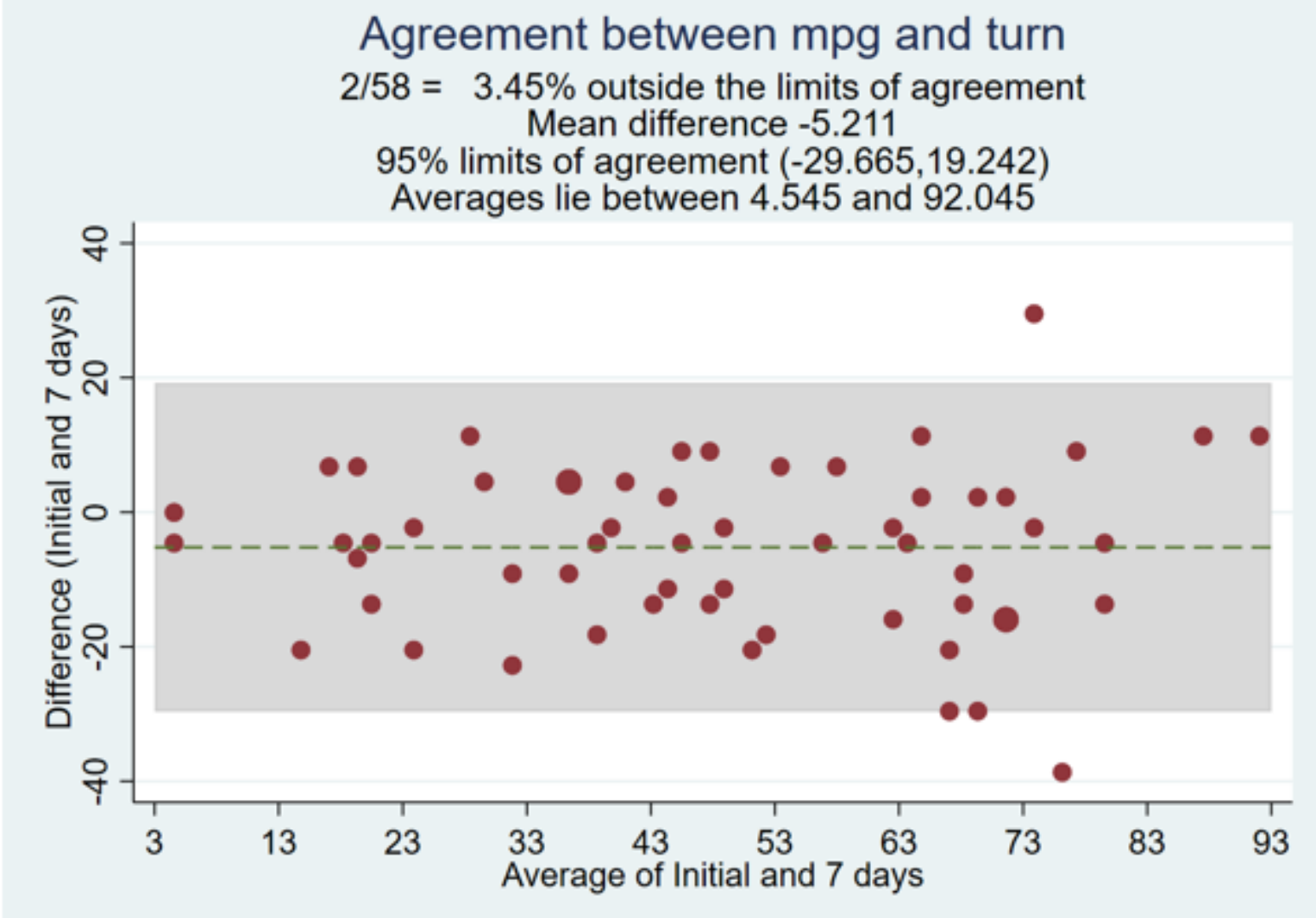

Figure 1

Bland-Altman plot for the agreement between test-retest measurements of the KOOS-PF. 


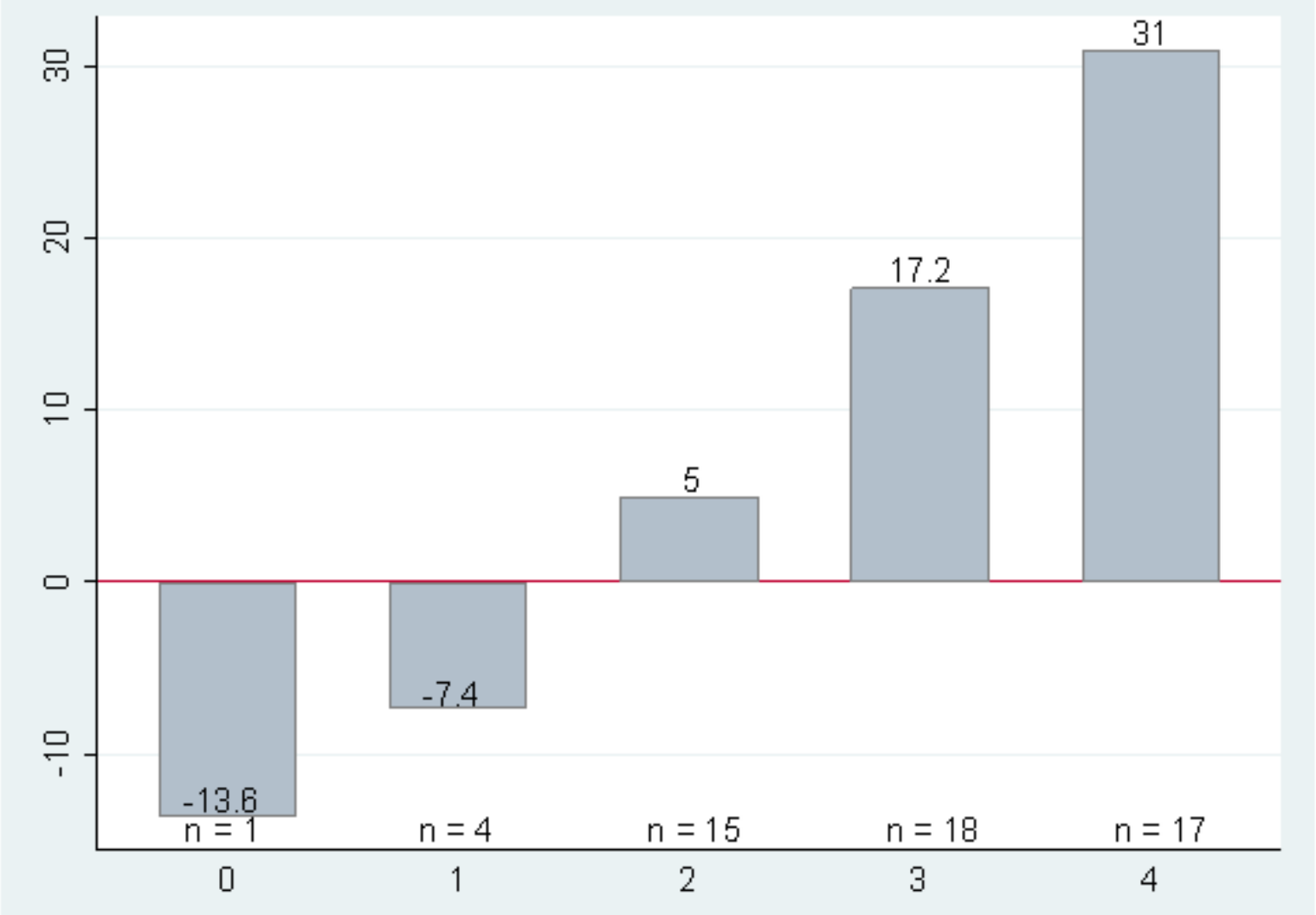

Figure 2

KOOS-PF mean change scores (negative change represents worsening) vs GROC scores ( $0=$ much worse; 1 = slightly worse; 2 = no change; 3 = slightly better; 4 = much better) demonstrated good correlation $(r=0.64)$. 


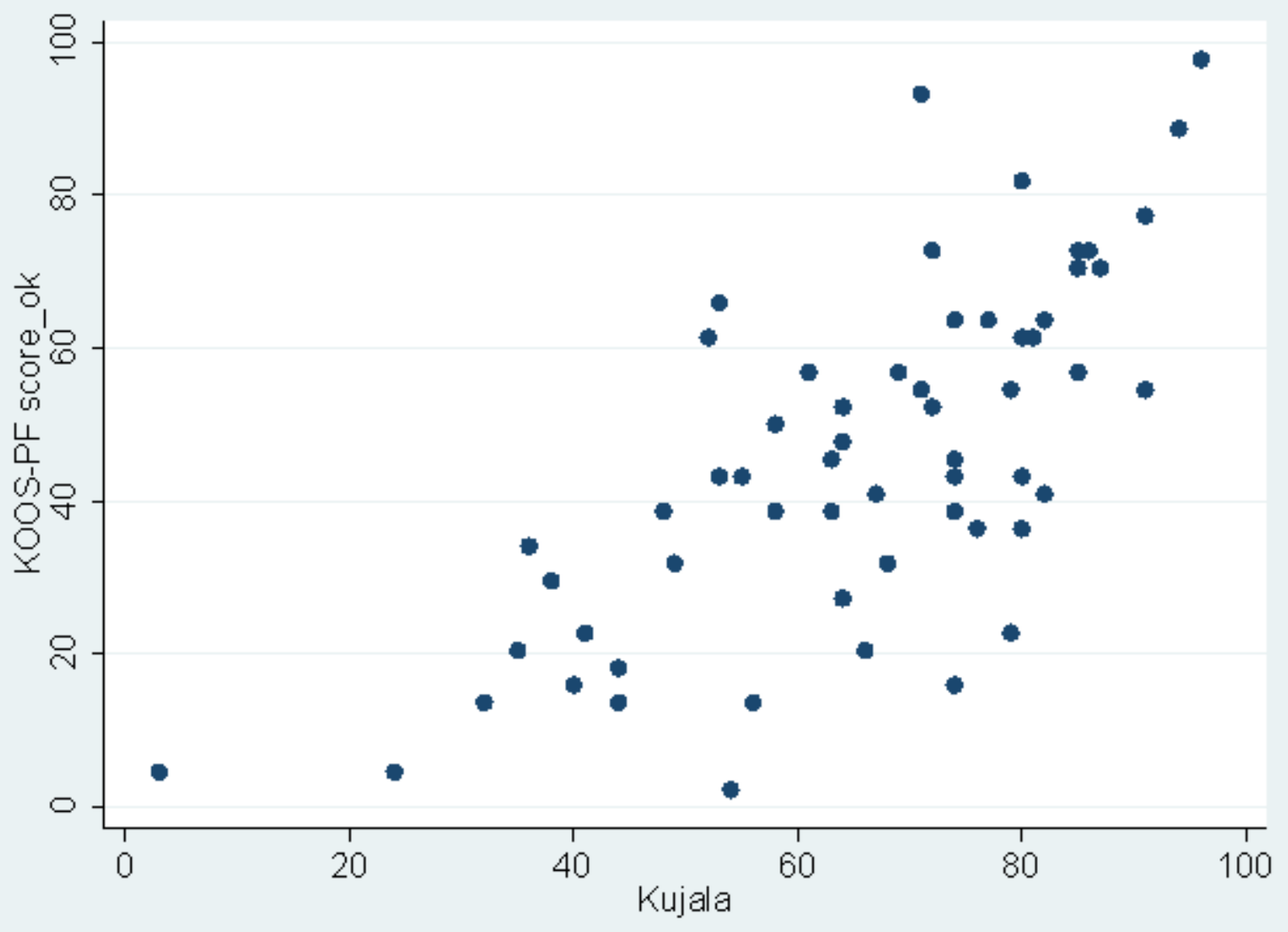

Figure 3

Scatter plot showing the correlation between the KOOS-PF subscale and the Kujala score, $r=0.71$. 